\title{
Four-Port Silicon Multi-Wavelength Optical Router for Photonic Networks-on-Chip
}

\author{
Ting $\mathrm{Hu}$, Haifeng Shao, Longzhi Yang, Chao Xu, Mei Yang, Hui Yu, Xiaoqing Jiang, and Jianyi Yang
}

\begin{abstract}
We design and fabricate a four-port wavelengthselective optical router on silicon-on-insulator wafer for photonic networks-on-chip. The router consists of four basic operation blocks. Each is constructed by one microring resonator (MRR) based add-drop filter rather than the traditional two microrings based $2 \times 2$ optical crossbar. It can provide multiwavelength routing for each path to increase the aggregate data transmission bandwidth. The possible $12 \mathrm{I} / \mathrm{O}$ routing paths are experimentally observed in the transmission spectra and each of them has the ability to route four group wavelengths simultaneously in the measured spectral range. The device has a small footprint $\left(\sim 78 \times 100 \mu \mathrm{m}^{2}\right)$ and low power consumption $(6.58 \mathrm{~mW})$.
\end{abstract}

Index Terms-Optical router, microring resonator, photonic networks-on-chip.

\section{INTRODUCTION}

$\mathbf{I}$ NTEGRATING a set of cores in the central processing unit, which is known as chip multiprocessors (CMPs), to realize high performance computing system is becoming the mainstream technology. As the feature size of the semiconductor manufacturing industry scales down, the metal line-based electrical interconnects between these cores face significant challenges to meet the CMPs' communication requirement due to their limited bandwidth, long time delay and high power consumption [1]. Compared with the traditional electrical interconnects, optical interconnects have the inherent advantages of higher bandwidth, lower latency and less power consumption [2], [3]. Hence, the photonic networks-on-chip (PNoC) used to improve the communication performance of the CMPs has attracted much research interests in recent years. Because the fabrication process is compatible with the complementary metal-oxide-semiconductor (CMOS) technology,

Manuscript received August 8, 2013; revised September 24, 2013; accepted October 3, 2013. Date of publication October 9, 2013; date of current version November 6, 2013. This work was supported in part by the Natural Science Foundation of China under Grant 61228501, in part by the 863 project under Grant 2012AA012203, in part by the Doctoral Discipline Foundation of the Ministry of Education under Grant 20120101110054, in part by the Award for Excellent Doctoral Student granted by the Ministry of Education of China, in part by the Science and Technology Innovation Projects for Students in Zhejiang Province under Grant 2013R401250, and in part by the U.S. National Science Foundation under Grant CNS-1126688.

T. Hu, H. Shao, L. Yang, C. Xu, H. Yu, X. Jiang, and J. Yang are with the Department of Information Science and Electronic Engineering, Zhejiang University, Hangzhou 310027, China (e-mail: huting@zju.edu.cn; zjushfly@zju.edu.cn; yanglongzhi61 @zju.edu.cn; xc@zju.edu.cn; huiyu@zju.edu.cn; iseejxq@zju.edu.cn; yangjy@zju.edu.cn).

M. Yang is with the Department of Electrical and Computer Engineering, University of Nevada, Las Vegas, NV 89154 USA (e-mail: mei.yang@unlv.edu).

Color versions of one or more of the figures in this letter are available online at http://ieeexplore.ieee.org.

Digital Object Identifier 10.1109/LPT.2013.2285171 silicon photonics is the most promising candidate to build the PNoC.

The optical routers, which are responsible for selecting the destinations for the input optical signals, play very important roles in PNoC. Silicon optical routers based on Mach-Zehnder interferometers (MZIs) and microring resonators (MRRs) have been demonstrated by previous works [4]-[11]. These routers can be divided to two types. One is constructed by active switches [4]-[8], which are controlled by electrical signals to establish the optical link paths before optical signal transmission. In these routers, the operating wavelength is uniform in all optical paths. The drawback is that they need additional switching time and power consumption for the optical path pre-configuring. The other one consists of passive add-drop ring filters [9]-[11]. All the paths between input and output ports are fixed and optical signals transmit with assigned wavelengths. Without regards to the rings' resonance shift induced by fabrication errors, these routers don't consume power to establish paths, but need multiple operating wavelengths. Fortunately, the recently reported work on silicon-based optical frequency comb source may be a promising solution to provide multiple working wavelengths simultaneously [12].

In this letter, we design and fabricate a four-port passive wavelength-selective optical router with the silicon-oninsulator (SOI) platform. Only four MRRs are used to implement the routers. Possible $12 \mathrm{I} / \mathrm{O}$ optical routing paths that can realize multi-wavelength routing are characterized by measuring the transmission spectra of them. Unlike the optical crossbar in [10] and [11], only three operating wavelengths are needed to realize communications among the four ports in our device and no paths exist for signals to enter and exit from the same port (U-turn). Compared to the router demonstrated in our previous work [9], this new design offers desirable optical spectra for multi-wavelength routing. Four group wavelengths (in the measured spectral range) with spacing equal to the MRRs' free spectral range (FSR) can be routed simultaneously to increase the aggregate bandwidth of the router, and the number of MRRs of this simple configuration is reduced by half, which leads to a smaller footprint and less power consumption.

\section{BASIC OPERATION ELEMENTS}

The microring resonator (MRR) based add-drop filter, built by two bus waveguides coupled to one ring waveguide, as shown in Fig.1 (a), is a natural $2 \times 2$ passive wavelengthselective optical crossbar (OCB). The input light signals launched to port $\mathrm{I}_{1}\left(\mathrm{I}_{2}\right)$ are coupled to port $\mathrm{O}_{1}\left(\mathrm{O}_{2}\right)$ if the 

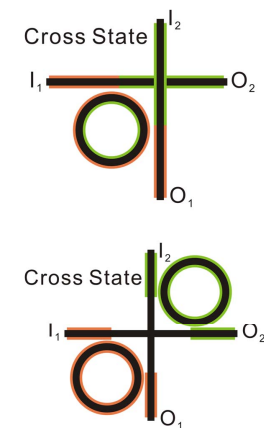

(a)
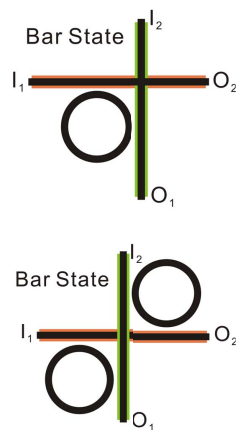

(b)

Fig. 1. (a) One MRR and (b) two MRRs based $2 \times 2$ wavelength-selective OCB.

input signals' wavelength $\lambda$ matches one of the resonance wavelengths of the MRR. This state is usually called as cross state. In other condition, the input signals fed to port $\mathrm{I}_{1}\left(\mathrm{I}_{2}\right)$ will simply transmit to port $\mathrm{O}_{2}\left(\mathrm{O}_{1}\right)$, which is the bar state Therefore, the destinations are different for the input signals with distinct wavelengths after they pass the OCB.

Signals that transmit from $\mathrm{I}_{2}$ to $\mathrm{O}_{2}$ need to pass the waveguide crossings twice while those from $\mathrm{I}_{1}$ to $\mathrm{O}_{1}$ do not pass any crossing. In order to reduce the insertion loss of the optical path $\mathrm{I}_{2}$ to $\mathrm{O}_{2}$, usually one more MRR is added near the crossing, as shown in Fig.1 (b). However there are some other problems in this two MRRs based $2 \times 2$ wavelength-selective OCB. For example, when optical signals are launched to $\mathrm{I}_{1}$ and match the MRRs' resonance condition, most optical power is dropped to port $\mathrm{O}_{1}$ through the first ring, but the residual optical power in the bus waveguide will form a feedback by the second ring, which will disturb the resonant modes in the first ring and lead the filter transmission spectra change. The optical power of $\mathrm{O}_{1}$ and $\mathrm{O}_{2}$ (normalized to $\mathrm{I}_{1}$ ) can be derived by the transfer matrix method [13]. The calculated results of the two $10-\mu \mathrm{m}$ radius MRRs based $2 \times 2$ OCB (with the SOI platform) are plotted in Fig. 2 (a). As seen from it, the transmission spectra present strong asymmetric property at most resonances More seriously, in the transmission spectrum of $\mathrm{O}_{1}$, large optical power dips exist near most resonances. The inset in Fig. 2 (a) shows the zoom-in spectra at the resonance near $1513 \mathrm{~nm}$. We can see a $\sim 5 \mathrm{~dB}$ intra-band power dip $50 \mathrm{pm}$ far away from the maximum power at $\mathrm{O}_{1}$. Since silicon is a temperature sensitive material with a large thermo-optic coefficient at $1550 \mathrm{~nm}$ [14] the MRR's resonance will shift about $50 \mathrm{pm}$ and lead to a $5 \mathrm{~dB}$ increase of the insertion loss of the path $\mathrm{I}_{1}-\mathrm{O}_{1}$ if the temperature fluctuates with $0.5 \mathrm{~K}$ The depth of the intra-band dips increase as the coupling between the ring and bus waveguides becomes stronger. This makes the device unable to realize multi-wavelength routing and not suitable to be used in PNoC. In the simulation, the waveguide's cross section and slab thickness are chosen to be $450 \times 220 \mathrm{~nm}^{2}$ and $60 \mathrm{~nm}$ for calculating the effective refractive index by using the finite element method. The electric amplitude coupling coefficient $\mathrm{k}$ and optical propagation loss (OPL) in the ring waveguide are assumed to be 0.2579 and $3 \mathrm{~dB} / \mathrm{cm}$.



(a)

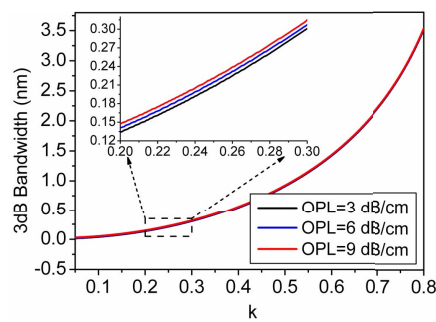

(c)

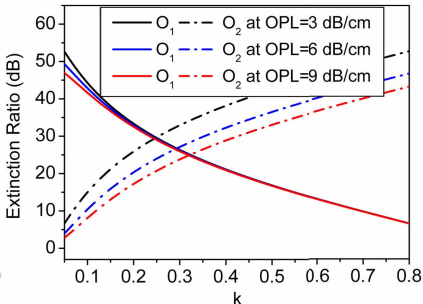

(b)

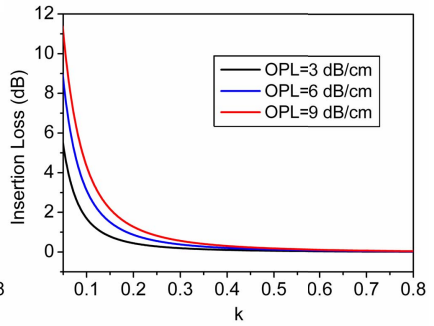

(d)
Fig. 2. (a) Calculated optical transmission spectra of the two MRRs based $2 \times 2$ wavelength-selective OCB. Calculated (b) ER, (c) $3 \mathrm{~dB}$ bandwidth and (d) insertion loss of the one MRR based $2 \times 2$ wavelength-selective OCB varying with $\mathrm{k}$ at different $\mathrm{OPL}$.

According to the analysis above, we choose the structure shown in Fig. 1 (a) as the basic operation block to build the four-port optical router. Its extinction ratio (ER), $3 \mathrm{~dB}$ bandwidth and insertion loss are plotted as a function of $\mathrm{k}$ in Fig. 2 (b)-(d). As seen, a lager OPL degrades the device's performance. For a given k, the increasing OPL decreases the ER and enlarges the $3 \mathrm{~dB}$ bandwidth and insertion loss. In this design, $\mathrm{k}$ is chosen around 0.26 to obtain a tradeoff among the ER of $\mathrm{O}_{1}$ and $\mathrm{O}_{2}$, bandwidth and insertion loss. The space between the ring and bus waveguides is determined about $250 \mathrm{~nm}$ to achieve $\mathrm{k}=0.26$ by the three dimension finite-difference time-domain (3D-FDTD) method.

\section{Design, Fabrication and Measurement OF THE $4 \times 4$ SILICON OPTICAL MULTI-WAVELENGTH ROUTER}

The schematic structure of the proposed $4 \times 4$ optical router is shown in Fig. 3 (a). The four ports are labeled as North (N), South (S), East (E), and West (W). Two groups of two parallel waveguides are used to connect $\mathrm{N}$ and $\mathrm{S}, \mathrm{W}$ and $\mathrm{E}$ directly. These groups of waveguides are designed intersecting vertically so that only one ring adjacent to the crossing is needed to establish the optical path between an input port and an output port. For example, $\mathrm{R}_{1}$ at the upper-left corner connects $\mathrm{N}_{\mathrm{i}}$ and $\mathrm{W}_{\mathrm{o}}$, while $\mathrm{R}_{3}$ at the bottom-left corner connects $\mathrm{N}_{\mathrm{i}}$ and $E_{0}$. Table 1 illustrates the possible optical paths of the router. As U-turn is not allowed, the diagonal entries in the table are blank. The 12 possible physical paths are presented with the assigned operating MRRs and wavelengths Some MRRs are using by two paths simultaneously, such as $R_{3}$ is both used by paths $\mathrm{N}_{\mathrm{i}}-\mathrm{E}_{\mathrm{o}}$ and $\mathrm{W}_{\mathrm{i}}-\mathrm{S}_{\mathrm{o}}$, but they will not lead the path to blocking because the two beams will propagate respectively though they interfere with each other in $\mathrm{R}_{3}$ 


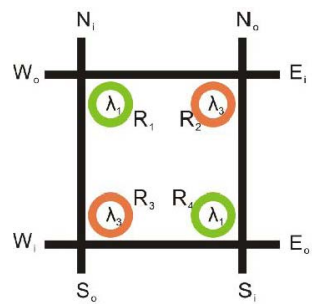

(a)



(b)
Fig. 3. (a) Schematic structure and (b) micrograph of the $4 \times 4$ multi-wavelength optical router.

TABLE I

Routing PATHS OF THE $4 \times 4$ OpticAl Router

\begin{tabular}{|c|c|c|c|c|c|}
\hline \multicolumn{2}{|c|}{} & \multicolumn{4}{|c|}{ Input } \\
\hline \multirow{3}{*}{} & & $\mathrm{N}_{\mathrm{i}}$ & $\mathrm{E}_{\mathrm{i}}$ & $\mathrm{W}_{\mathrm{i}}$ & $\mathrm{S}_{\mathrm{i}}$ \\
\cline { 2 - 6 } & $\mathrm{N}_{0}$ & - & $\mathrm{R}_{2}\left(\lambda_{3}\right)$ & $\mathrm{R}_{4}\left(\lambda_{1}\right)$ & None $\left(\lambda_{2}\right)$ \\
\cline { 2 - 6 } & $\mathrm{E}_{0}$ & $\mathrm{R}_{3}\left(\lambda_{3}\right)$ & - & None $\left(\lambda_{2}\right)$ & $\mathrm{R}_{4}\left(\lambda_{1}\right)$ \\
\cline { 2 - 6 } & $\mathrm{W}$ & $\mathrm{R}_{1}\left(\lambda_{1}\right)$ & None $\left(\lambda_{2}\right)$ & - & $\mathrm{R}_{2}\left(\lambda_{3}\right)$ \\
\cline { 2 - 6 } & $\mathrm{S}_{0}$ & None $\left(\lambda_{2}\right)$ & $\mathrm{R}_{1}\left(\lambda_{1}\right)$ & $\mathrm{R}_{3}\left(\lambda_{3}\right)$ & - \\
\hline
\end{tabular}

The router is fabricated on an 8-inch SOI wafer with 220-nm-thick top Si layer and 2- $\mu \mathrm{m}$-thick buried dioxide layer. The fabrication process is the same with that in [15], [16]. Rib waveguides with a cross section of $450 \times 220 \mathrm{~nm}^{2}$ and a slab of 60-nm thick are adopted to only support fundamental quasi-transverse-electric (quasi-TE) mode. Gaps between the bus and ring waveguides are selected to be $250 \mathrm{~nm}$ according to the simulation above. The radii of $\mathrm{R}_{1}$ and $\mathrm{R}_{4}, \mathrm{R}_{2}$ and $\mathrm{R}_{3}$ are chosen to be $10 \mu \mathrm{m}$ and $10.01 \mu \mathrm{m}$, respectively, to obtain two different resonance wavelengths $\left(\lambda_{1}, \lambda_{3}\right)$ with the spacing around $1.6 \mathrm{~nm}(200 \mathrm{GHz})$, which matches International Telecommunication Unit grid for WDM systems. TiN heaters are made to compensate the MRRs' resonance shift caused by fabrication errors. Optimized ellipse-based waveguide crossings, with the semi-major axis and semi-minor axis of 4 and $1 \mu \mathrm{m}$, respectively, are utilized to reduce the OPL. The insertion loss of the crossing waveguide is measured around $0.61 \mathrm{~dB} /$ crossing by the cut-off method. Grating couplers are integrated to couple light into and out of the device. The topview optical micrograph of a typical fabricated device is shown in Fig. 3 (b).

The fabricated device is characterized by using an amplified spontaneous emission (ASE) source and an optical spectrum analyzer (OSA). The broadband light is coupled into each input port $\left(\mathrm{N}_{\mathrm{i}}, \mathrm{S}_{\mathrm{i}}, \mathrm{W}_{\mathrm{i}}, \mathrm{E}_{\mathrm{i}}\right)$ and collected at each output port $\left(\mathrm{N}_{\mathrm{o}}, \mathrm{S}_{\mathrm{o}}, \mathrm{W}_{\mathrm{o}}, \mathrm{E}_{\mathrm{o}}\right)$, and then fed into the OSA. A total electric power consumption of $6.58 \mathrm{~mW}$ is used to drive the TiN heaters for resonances adjusting. Fig 4 shows the measured TE-polarized (electric field parallel to the chip) transmission spectra for the $12 \mathrm{I} / \mathrm{O}$ optical routing paths. The spectra of the four U-turn paths are also presented in Fig. 4 for completeness. We can see from the figure that when the signals with the wavelengths of $\lambda_{1}, \lambda_{2}$ and $\lambda_{3}$ (wavelength interval is equal to $0.8 \mathrm{~nm}$ ) are injected into one of the four input ports, they will be routed to the other three output ports. For instance, if the signals with the wavelengths of $\lambda_{1}, \lambda_{2}$ and $\lambda_{3}$ are launched into $E_{i}$, they will be guided to $S_{0}, W_{0}$

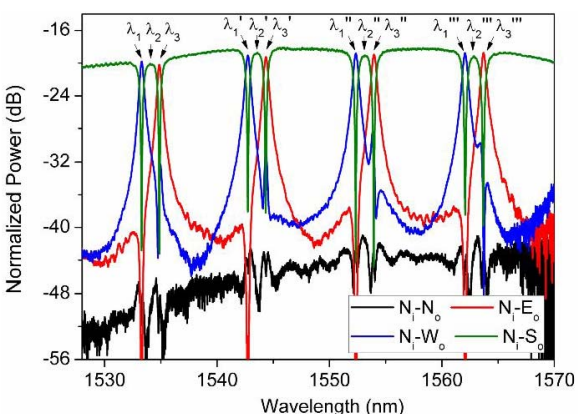

(a)

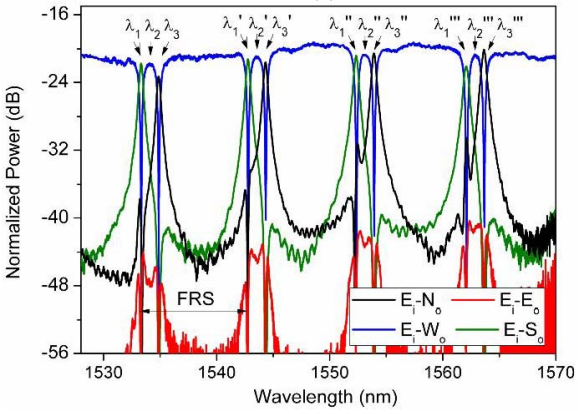

(b)



(c)

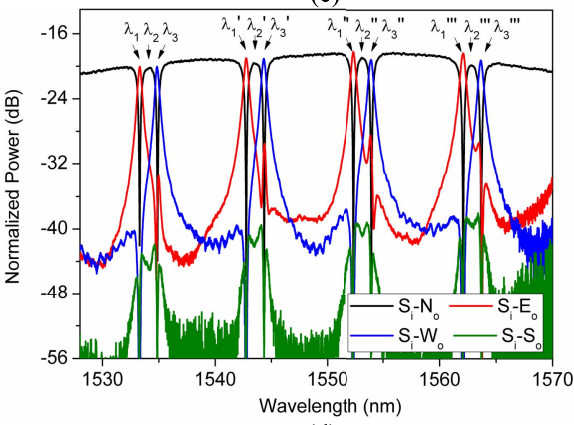

(d)

Fig. 4. Measured optical transmission spectra at output ports of the $4 \times 4$ multi-wavelength router when broadband light is injected into port (a) $\mathrm{N}_{i}$, (b) $\mathrm{E}_{i}$, (c) $\mathrm{W}_{i}$ and (d) $\mathrm{S}_{i}$, respectively.

and $\mathrm{N}_{\mathrm{o}}$, respectively. The common $3 \mathrm{~dB}$ bandwidth of these resonances is around $0.48 \mathrm{~nm}$, which is very narrow compared to the MZIs-based optical routers [5], [8]. However, we can utilize all the wavelengths whose interval is equal to the MRRs' FSR to realize the multi-wavelength routing, which mitigates the limitation and increases the aggregate bandwidth of the router. As seen in Fig. 4, four group wavelengths $\left(\lambda_{1}\right.$, $\left.\lambda_{2}, \lambda_{3} ; \lambda_{1}{ }^{\prime}, \lambda_{2}, \lambda_{3}{ }^{\prime} ; \lambda_{1} ", \lambda_{2} ", \lambda_{3}{ }^{\prime} ; \lambda_{1}{ }^{\prime \prime}, \lambda_{2} ",, \lambda_{3} " '\right)$ can be used for each path routing during the measured spectral range from $1528 \mathrm{~nm}$ to $1570 \mathrm{~nm}$. Since the FSR is inversely proportional to the MRR' radius, more operating wavelengths 
TABLE II

EXPERIMENTAL CT OF THE ROUTER WITH OPERATING WAVELENGTHS OF $\lambda_{1}^{\prime}, \lambda_{2}^{\prime}$, AND $\lambda_{3}^{\prime}$

\begin{tabular}{|c|c|c|c|c|}
\hline Paths $\mathrm{CT}(\mathrm{dB})$ & $\mathrm{CT}$ to $\mathrm{N}_{\mathrm{o}}$ & CT to $E_{o}$ & $\mathrm{CT}$ to $\mathrm{W}_{\mathrm{o}}$ & CT to $S_{o}$ \\
\hline $\mathrm{N}_{\mathrm{i}}-\mathrm{E}_{\mathrm{o}}$ & -25.98 & - & -11.31 & -18.93 \\
\hline $\mathrm{N}_{\mathrm{i}}-\mathrm{W}_{\mathrm{o}}$ & -25.58 & $<-36.59$ & - & -19.7 \\
\hline $\mathrm{N}_{\mathrm{i}}-\mathrm{S}_{\mathrm{o}}$ & -29.96 & -12.69 & -11.41 & - \\
\hline $\mathrm{E}_{\mathrm{i}}-\mathrm{N}_{\mathrm{o}}$ & & -31.552 & -18.6 & $<-34.66$ \\
\hline $\mathrm{E}_{\mathrm{i}}-\mathrm{W}_{\mathrm{o}}$ & -11.21 & -23.93 & - & -11.17 \\
\hline $\mathrm{E}_{\mathrm{i}}-\mathrm{S}_{\mathrm{o}}$ & -27.15 & $<-35.97$ & -25.54 & - \\
\hline $\mathrm{W}_{\mathrm{i}-\mathrm{N}_{\mathrm{o}}}$ & & -20.63 & $<-38.23$ & $\begin{array}{l}-28.643 \\
\end{array}$ \\
\hline $\mathrm{W}_{\mathrm{i}}-\mathrm{E}_{\mathrm{o}}$ & -12.41 & - & -25.58 & -10.54 \\
\hline $\mathrm{W}_{\mathrm{i}-}-\mathrm{S}_{\mathrm{o}}$ & $<-37.72$ & -23.69 & -36.5 & - \\
\hline $\mathrm{S}_{\mathrm{i}-\mathrm{N}_{\mathrm{o}}}$ & - & -10.07 & -10.77 & -22.1 \\
\hline $\mathrm{S}_{\mathrm{i}}-\mathrm{E}_{\mathrm{o}}$ & -26.78 & - & $<-37.24$ & -33.01 \\
\hline $\mathrm{S}_{\mathrm{i}-\mathrm{W}_{\mathrm{o}}}$ & -26.39 & -11.42 & - & -36.81 \\
\hline
\end{tabular}

can be utilized for each path and the bandwidth utilization efficiency will be further increased by using MRRs with larger radius. From the measured transmission spectra, the crosstalk (CT) can be calculated as the difference between the power at destination signal and that leaks to other output ports. The CT of the second group operating wavelengths $\left(\lambda_{1}{ }^{\prime}, \lambda_{2}{ }^{\prime}, \lambda_{3}{ }^{\prime}\right)$ is presented in Table 2 . As seen, the optical paths working with $\lambda_{2}$, i.e. $\mathrm{N}_{\mathrm{i}}-\mathrm{S}_{\mathrm{o}}, \mathrm{E}_{\mathrm{i}}-\mathrm{W}_{\mathrm{o}}, \mathrm{W}_{\mathrm{i}}-\mathrm{S}_{\mathrm{o}}$ and $\mathrm{S}_{\mathrm{i}}-\mathrm{N}_{\mathrm{o}}$, generate a relative worse $\mathrm{CT}$ (from -10.07 to $-12.69 \mathrm{~dB}$ ). Adopting high order MRR-based filter with box-like transmission spectra can improve this performance [15]. In Fig. 4(a), the optical spectrum of $\mathrm{N}_{\mathrm{i}}-\mathrm{E}_{\mathrm{o}}$ has a ripple at $\lambda_{3}, \lambda_{3}, \lambda_{3}$ "and $\lambda_{3}$ ", which may deteriorate the $\mathrm{CT}$ to port $\mathrm{W}_{\mathrm{o}}$, is caused by the interference of the crossing-induced $\mathrm{CT}$ and $\mathrm{R}_{1}$-induced $\mathrm{CT}$ at these wavelengths. Similar phenomena of $\mathrm{E}_{\mathrm{i}}-\mathrm{N}_{\mathrm{o}}, \mathrm{W}_{\mathrm{i}}-\mathrm{S}_{\mathrm{o}}$ and $\mathrm{S}_{\mathrm{i}}-\mathrm{E}_{\mathrm{O}}$ are observed in Fig. 4(b)-(d), respectively. This problem can be solved by utilizing the MMI-based crossing with low CT [17]. All these further optimizations are under consideration in our future works.

\section{CONCLUSION}

In conclusion, we design and fabricate a four-port wavelength-selective optical router on SOI platform by CMOS compatible process. The device is constructed by only four MRRs with the footprint around $78 \times 100 \mu \mathrm{m}^{2}$ (not including the pads). Possible 12 I/O optical paths that can implement multi-wavelength routing are observed in the measured transmission spectra by utilizing $6.58 \mathrm{~mW}$ electric power consumption to compensate the MRRs' resonance wavelength shift caused by fabrication errors. This multi-wavelength routing method has the ability to increase the aggregate data transmission bandwith. The CT of the router can be improved by further device optimizations. If the post trimming process is employed to adjust the MRRs' resonance wavelengths [18], such wavelength-selective routers can work with ultra low power consumption in the practical system, which is only used to compensate the resonance wavelength shift induced by the environmental temperature fluctuation and eliminate the misalignment between the light source and resonance wavelengths. These advantages lead them potential to apply in $\mathrm{PNoC}$.

\section{REFERENCES}

[1] R. G. Beausoleil, P. J. Kuekes, G. S. Snider, S. Wang, and R. S. Williams, "Nanoelectronic and nanophotonic interconnect," Proc. IEEE, vol. 96, no. 2, pp. 230-247, Feb. 2008.

[2] F. Benner, M. Ignatowski, J. A. Kash, D. M. Kuchta, and M. B. Ritter, "Exploitation of optical interconnects in future server architectures," IBM J. Res. Develop., vol. 49, nos. 4-5, pp. 755-775, Jul. 2005.

[3] A. Shacham, K. Bergman, and L. P. Carloni, "Photonic networks-on-chip for future generations of chip multiprocessors," IEEE Trans. Comput., vol. 57, no. 9, pp. 1246-1260, Sep. 2008.

[4] N. Sherwood-Droz, et al., "Optical $4 \times 4$ hitless slicon router for optical networks-on-chip (NoC)," Opt. Express, vol. 16, no. 20, pp. 15915-15922, Sep. 2008.

[5] M. Yang, et al., "Non-blocking $4 \times 4$ electro-optic silicon switch for on-chip photonic networks," Opt. Express, vol. 19, no. 1, pp. 47-54, Jan. 2011.

[6] R. Ji, J. Xu, and L. Yang, "Five-port optical router based on microring switches for photonic networks-on-chip," IEEE Photon. Technol. Lett., vol. 25, no. 5, pp. 492-494, Feb. 18, 2013.

[7] R. Min, R. Ji, Q. Chen, L. Zhang, and L. Yang, "A universal method for constructing $\mathrm{N}$-port nonblocking optical router for photonic networks-on-chip," J. Lightw. Technol., vol. 30, no. 23, pp. 3736-3741, Dec. 1,2012

[8] X. Li, et al., "Mach-Zehnder-based five-port silicon router for optical interconnects," Opt. Lett., vol. 38, no. 10, pp. 1703-1705, May 2013.

[9] T. Hu, et al., "Wavelength-selective $4 \times 4$ nonblocking silicon optical router for networks-on-chip," Opt. Lett., vol. 36, no. 23, pp. 4710-4712, Dec. 2011.

[10] L. Zhou, et al., "Design and evaluation of an arbitration-free passive optical crossbar for on-chip interconnection networks," Appl. Phys. A, vol. 95, no. 4, pp. 1111-1118, Jun. 2009.

[11] A. Kazmierczak, et al., "Highly integrated optical $4 \times 4$ crossbar in silicon-on-insulator technology," J. Lightw. Technol., vol. 27, no. 16, pp. 3317-3323, Aug. 15, 2009

[12] M. A. Foster, J. S. Levy, O. Kuzucu, K. Saha, M. Lipson, and A. L. Gaeta, "Silicon-based monolithic optical frequency comb source," Opt. Express, vol. 19, no. 15, pp. 14233-14239, Jun. 2011.

[13] A. Yariv, "Universal relations for coupling of optical power between microresonators and dielectric waveguides," Electron. Lett., vol. 36, no. 4, pp. 321-322, Feb. 2000.

[14] J. Komma, C. Schwarz, G. Hofmann, D. Heinert, and R. Nawrodt, "Thermo-optic coefficient of silicon at $1550 \mathrm{~nm}$ and cryogenic temperatures," Appl. Phys. Lett., vol. 101, no. 4, pp. 041905-1-041905-4, Jul. 2012.

[15] T. Hu, et al., "Thermally tunable filters based on third-order microring resonators for WDM applications," IEEE Photon. Technol. Lett., vol. 24 no. 6, pp. 524-526, Mar. 15, 2012.

[16] T. Hu, et al., "Tunable Fano resonances based on two-beam interference in microring resonator," Appl. Phys. Lett., vol. 102, no. 1, pp. 011112-1-011112-4, Jan. 2013.

[17] H. Chen and A. W. Poon, "Low-loss multimode-interference-based crossings for silicon wire waveguides," IEEE Photon. Technol. Lett., vol. 18 , no. 21, pp. 2260-2262, Nov. 1, 2006.

[18] A. H. Atabaki, A. A. Eftekhar, M. Askari, and A. Adibi, "Accurate post-fabrication trimming of ultra-compact resonators on silicon," Opt. Express, vol. 21, no. 12, pp. 14139-14145, Jun. 2013. 